\title{
Vibro-impact response of the cracked structures to monitor structural health
}

\author{
V. Babitsky, V. Hiwarkar, and V. Silberschmidt \\ Wolfson School of Mechanical and Manufacturing Engineering, Loughborough University, Loughborough, \\ Leicestershire, LE11 3TU, UK
}

\begin{abstract}
A method of simulation is developed for studying the dynamics of the structures with discontinuities using Matlab-Simulink. Simulation and experiments were performed to analyse the nonlinear behaviour of the cracked bar. It is found that the crack-induced nonlinearity leads to the generation of higher harmonics along with side band frequencies. Their intensity diminishes with increase in distance of measurement from the crack. The presence of sideband frequencies indicates modulation of exciting frequency due to systematic interaction of crack faces. The nonlinear transformation of modulated vibration by crack leads to generation of a low frequency periodic component. Its amplitude is proportional to the forced response of the cracked bar at the exciting frequency. The phenomenology described here can be effective for Structural Health Monitoring (SHM).
\end{abstract}

\section{Introduction}

The presence of a crack in the structure can drastically change the general dynamic characteristics of the structure, which results in the excitation of significant additional perturbations. The latter is mainly due to generation of intensive forces of impact and friction between the contact surfaces of the discontinuous elements. Few researchers have tried to address the issue of effect of the crack as discontinuity on the dynamics of the structure [1]. The main specific of the current research is concentrate on the vibro-impact response of the cracked structure. Vibro-impact resonances in structures with discontinuities were previously studied in [2].

The new simulation technique has been developed in the paper to study the effect of crack on the dynamics of the structure. Implantation of discontinuous elements like crack into a continuous structure needs a special mathematical technique for proper matching of local and distributed elements of the resulting structure. This can be based on the use of the force characteristics of contact interaction for the discontinuous elements and Green's function calculated in the contact areas for the media. This description leads to application of integral equation [2]. Laplace transformation of the integral equation produce operators for the following simulation of the interaction, where local discontinuity is implanted as a nonlinear feedback and continuous elements as the operators of dynamic compliance (receptance) of the media at the contact points.

Effective numerical simulation of such structures with Matlab-Simulink software needs a development of realistic models for contact phenomena and transformation of the transcendental operators of dynamic compliances into rational functions of complex variable. The latter can be made with the help of modal representation for the dynamic compliances [3]. Uniform convergence of the modal approximation leads to high accuracy of simulation within the use of first few modes. As an example, a model of the one-dimensional cracked cantilever bar subjected to longitudinal harmonic excitation is used to analyse a vibro-impact response as a way to monitor the structural health. The nonlinear phenomena due to vibro-impact interaction within the cracked bar are obtained and analysed.

\section{Problem formulation}

Consider a cantilever bar of length $l$ fixed at both ends with a plane cross-sectional area $S$ having modulus of elasticity $E$, mass density $\rho$ and excited by the harmonic force $P(x, t)=a_{p} \cos \omega t$ at the distance $\boldsymbol{x}$ from the left end of the bar (Fig.1(a)). Introduction of crack in the fixed -fixed bar at the right end splits the system into two subsystems (Fig.1(b)). One of the subsystems is the cantilever bar fixed at the left end only and other 
subsystem is the crack modelled as a limiter. For the convenience of the calculations the harmonic force $P(l, t)$ is applied at the free end of the cantilever bar which is responsible for cracks faces interaction. This interaction of the bar with

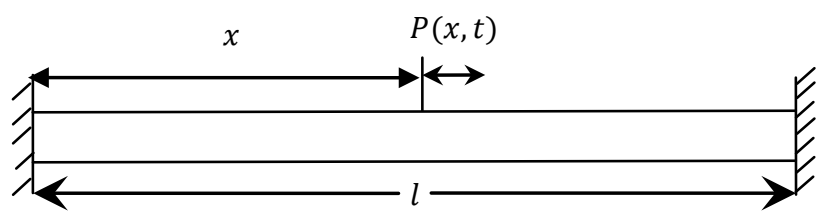

(a) Un-cracked

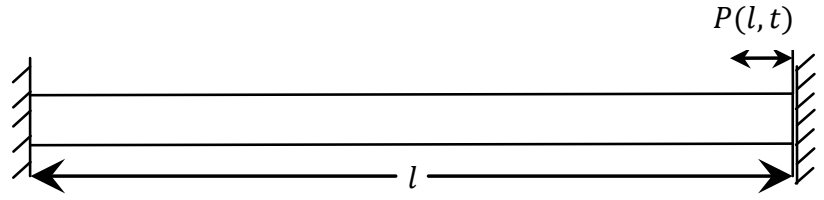

(b) Cracked Bar

Fig.1. Schematic of un-cracked and cracked bar

the limiter (considered as a crack in this case) leads to generation of the nonlinear contact force due to opening and closing of the crack. The blocks of the receptance and the contact force were modelled with MatlabSimulink.

The differential equation and boundary conditions for the longitudinal vibration of a cantilever bar are:

$\frac{\partial^{2} u(x, t)}{\partial t^{2}}-\gamma^{2} \frac{\partial^{2} u(x, t)}{\partial x^{2}}-b \frac{\partial^{3} u(x, t)}{\partial x^{2} \partial t}=0$

$u(0, t)=0, E S\left(1+\frac{b}{\gamma^{2}}\right)\left[\frac{\partial(x, t)}{\partial x}\right]_{x=l}=\exp (j \omega t)$

where

$\gamma=\sqrt{\mathrm{E} / \rho} \quad b=\gamma^{2} \chi / 2 \pi \omega$

$\omega$ is the frequency of excitation; $\chi$ is the absorption coefficient found from the test as the ratio of energy absorption during the cycle (proportional to the hysteresis loop) to the basic deformation energy.

Following [2], the expression for the receptance can be derived by applying the boundary condition of the cantilever bar to the Eq. (1) which is as follows:

$L_{l}(l, j \omega)=\frac{l}{E S \zeta}\left[\tan \zeta-j \frac{\chi}{4 \pi} \frac{\zeta+1 / 2 \sin 2 \zeta}{\cos ^{2} \zeta}\right]$

where

$\zeta=\frac{\omega l}{\gamma}$

The closed form of the receptance in Eq. (2) cannot be implemented numerically. For straight rods undergoing axial vibration this can be done by splitting it into infinite number of modes [2,3]. The receptance of the cross section at $x=p$ under the force applied to cross section $x=q$ for the straight elastic rods undergoing axial vibration can be written as :

$L_{p q}(s)=\sum_{v=1}^{\infty} \frac{A_{v}(p) A_{v}(q)}{s^{2}+2 r_{v} \Omega_{v} s+\Omega_{v}^{2}}$

in which $\mathrm{s}$ is a complex variable. The mechanism of energy dissipation in equation (3) is represented in the form of internal friction in the material, given by $\mathrm{r}_{\mathrm{v}}=$ $\frac{\chi \Omega_{\mathrm{v}}}{4 \pi \omega}$. Here $\Omega_{\mathrm{v}}$ represents the natural frequency and $\omega$ is the frequency of excitation. The number of vibration modes in Eq. (3) increases infinitely and the coefficients for each mode remains functions of continuous coordinate $x$, which represents the position of section under consideration; these coefficients are denoted as $A_{v}(x)$. It is also called the modal shape functions.

\section{Characteristics of contact force}

Under harmonic loading the bar interacts with the limiter (Fig.1(b)). This interaction with the limiter transforms the continuous process into successive impulses modulated by the velocity of the input process at the instant when the process reaches the threshold value. Fig.2 shows an example of temporal transformations of deformation $u(x, t)$ into the force $F[u(x, t)]$ by impact element with force characteristic $F(u)$.

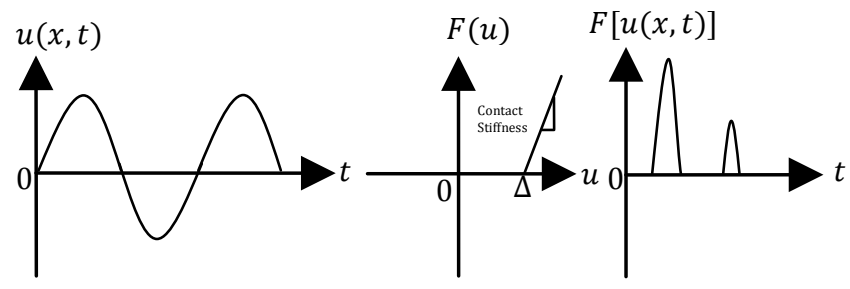

Fig. 2. Contact force characteristics

This force characteristic of the contact interaction is given by an expression called static force characteristics of the impact pair [2]:

$F(u)=\psi(u-\Delta) \eta(u-\Delta)$

Where

$\psi$ is a linearised coefficient of contact stiffness $\eta(u)$ is Heaviside's unit step function.

\section{Dynamic model of cracked cantilever bar}

From the schematic shown in Fig.1 (b) it is clear that when the harmonic loading is acting on the bar it starts to interact with a limiter, generating the contact force that affects deformation of the bar. The schematic shown in Fig. 1(b) can be represented in the block diagram shown in Fig.3, which is used for the analysis purpose. 


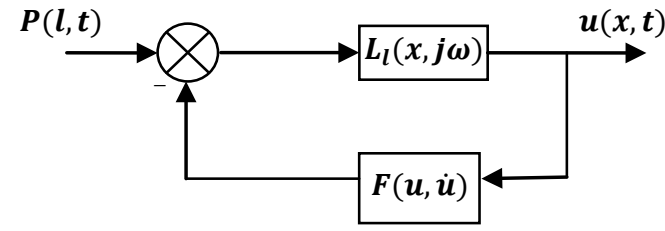

Fig.3. Block diagram

Using operator $L_{l}(x, j \omega)$ which connects the displacement of element $x$ due to an arbitrary applied force applied to an element $l$, coupling displacement $u(x, t)$ to the force acting at $x=l$, the operator equation for the unknown function $u(x, t)$ can be written in the following form [2]:

$u(x, t)=L_{l}(x, s) P(l, t)-L_{l}(x, s) F(u, \dot{u})$

The simulation with Matlab-Simulink is performed on the cantilever bar taking Eq. (4) and Eq. (5) into account. For simulation purpose only first four modes of vibration are taken into consideration which gives the accurate numerical results. For simulations the material properties of steel rod are considered having dimensions $300 \mathrm{~mm} \times 25 \mathrm{~mm} \times 10 \mathrm{~mm}$.

\section{Response of the cantilever bar}

Initially, the forced longitudinal vibration was performed on a cantilever bar without crack. The frequency sweep was performed in the Matlab-Simulink model with frequencies ranging from $3500 \mathrm{~Hz}$ to $35000 \mathrm{~Hz}$. The

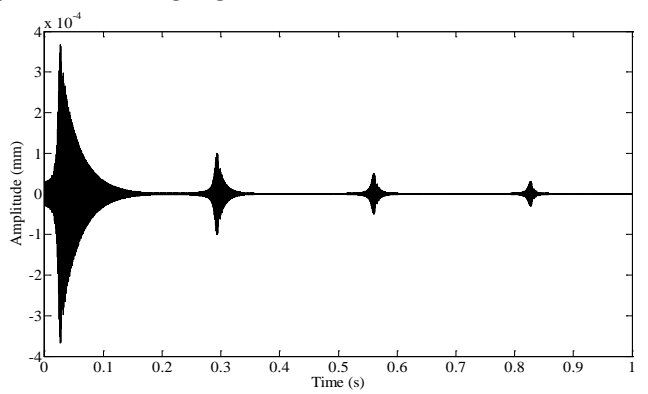

(a) Time response

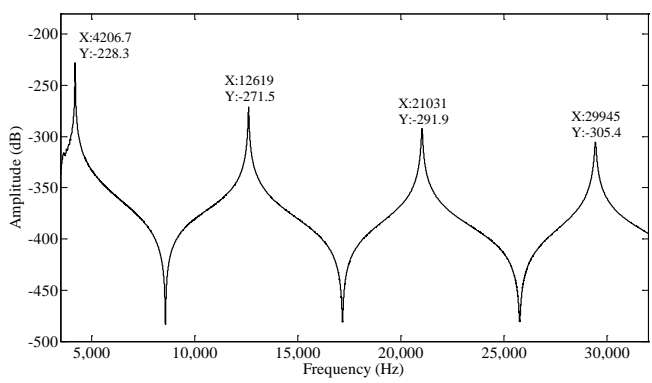

(b) Frequency response

Fig. 4. Response of cantilever bar (linear case) results in Fig. 4(a) and Fig. 4(b) show the time and frequency response, respectively, of the cantilever bar. From the time response it can be observed that it is symmetric about the x-axis. The frequency response of the system clearly shows that the resonance occurring at the frequencies of $4206 \mathrm{~Hz}, 12620 \mathrm{~Hz}, 21029 \mathrm{~Hz}$ and $29945 \mathrm{~Hz}$.

Similarly, for a cantilever bar with a crack, the frequency sweep was performed from $3500 \mathrm{~Hz}$ to 35000 $\mathrm{Hz}$ in the Matlab-Simulink model. The result in Fig. 5(a) and Fig. 5(b) shows the time response and frequency response, respectively. From the time response it can be observed that the time signal has lost its symmetry, indicating distortion. This distortion in time signal is an indication of nonlinearity due to opening and closing of the crack. In frequency response (Fig.5 (b)) it can be observed that there has been shift in the frequency when compared with the frequency response of the uncracked bar (Fig.4 (b)) along with higher harmonic frequency components occurring in the frequency response indicating the crack-induced nonlinearity.

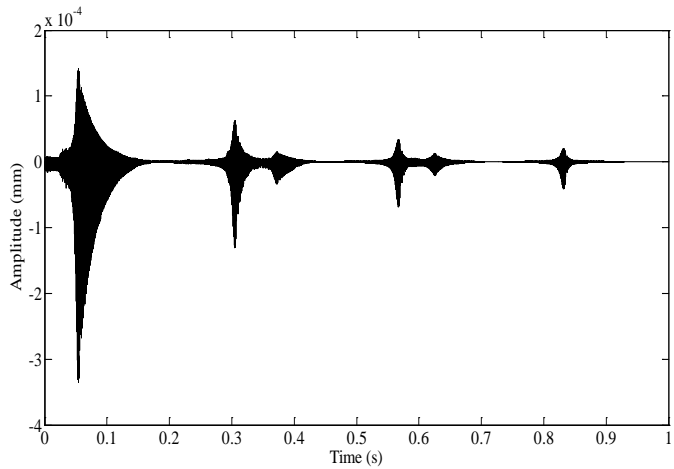

(a) Time response

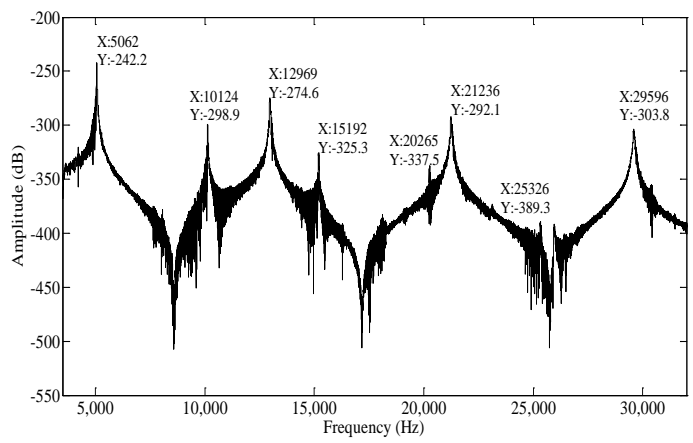

(b) Frequency response

Fig. 5. Response of cracked cantilever bar (nonlinear case)

\section{Effect of linear resonance frequency on cracked bar}

For the cantilever bar without a crack the first four resonant frequencies occurs at $4206 \mathrm{~Hz}, 12620 \mathrm{~Hz}, 21029$ $\mathrm{Hz}$ and $29945 \mathrm{~Hz}$. The cracked bar was excited at each of 
these resonant frequencies to obtain the steady-state response; the results for the first resonant frequency are presented in Fig.6. It can be observed that arrival of crack leads to interaction of two main periodic components corresponding to linear and nonlinear resonant frequencies. As a result the time response of the bar has modulation (Fig 6(a)). The nonlinear transformation of modulated signal by crack detects a low frequency component which frequency is difference of two high frequencies mentioned. It is useful to emphasise that the frequency of the low frequency component generated by cracked bar does not exist in the spectra of the bar's high frequency excitation and has no simple relationship with the frequency of excitation. Arrival of this component can be a good diagnostic feature for tracing of crack in the structure. So the crack behaves like a modulator and a detector of the low frequency component.

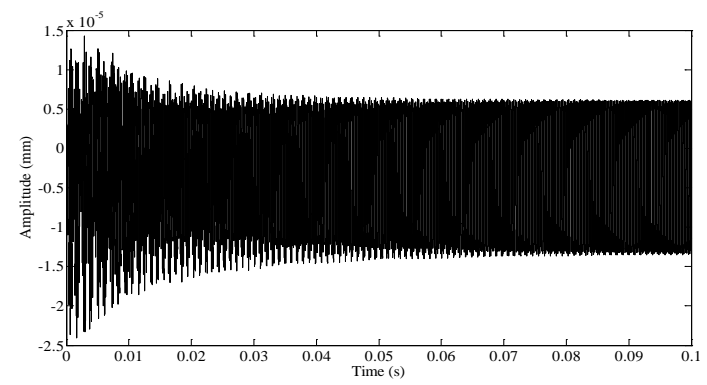

(a) Time response

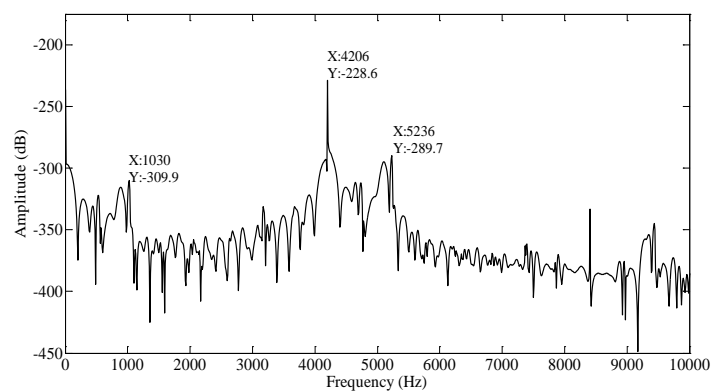

(b) Frequency response

Fig.6. Response at first linear resonance frequency of excitation

\section{Influence of crack on intensity of higher harmonics along bar}

The cracked bar was excited at the resonance frequencies of uncracked bar to obtain the steady-state response at each of this frequency. The measurement of the response is taken at the different points on the bar, i.e. changing the distance from the crack. It was observed from the frequency response that as one moves away from the crack, the effect of nonlinearity decreases as shown for the first resonant frequency of excitation (Fig.7). This indicates that the crack-induced nonlinearity is localized phenomenon. It can also be observed from the frequency responses that the magnitude of low frequency component is proportional to the magnitude of bar response at the excitation frequency. This indicates that crack behaves as an amplifier at bar resonant frequencies.

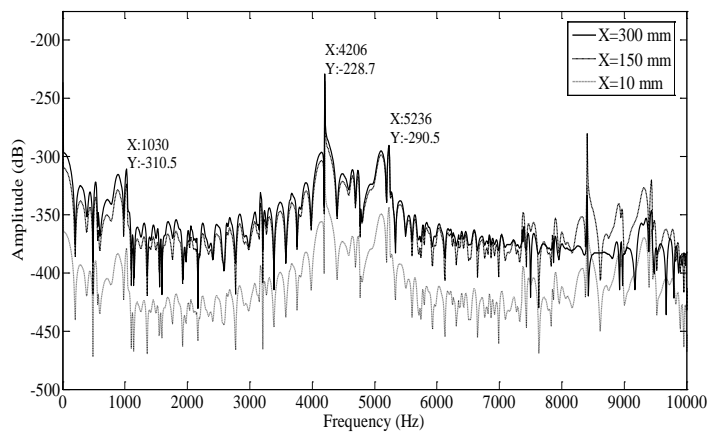

Fig.7. Influence of measurement point from the crack on higher harmonics

\section{Experiments}

A aluminium bar of $300 \mathrm{~mm} \mathrm{x} 25 \mathrm{~mm} \mathrm{x} 10 \mathrm{~mm}$ dimensions with fatigue crack at the centre, shown in Fig.8, was used in experiment. A small notch of $1 \mathrm{~mm}$ depth was made in the aluminium specimen and the fatigue crack was generated using the Instron Testing machine. This aluminium bar was fixed at the left end and the other end of bar was excited by the harmonic loading using the shaker which was driven by the oscillator. The piezo-strain gauges were glued at four different locations on the cracked bar for measuring a

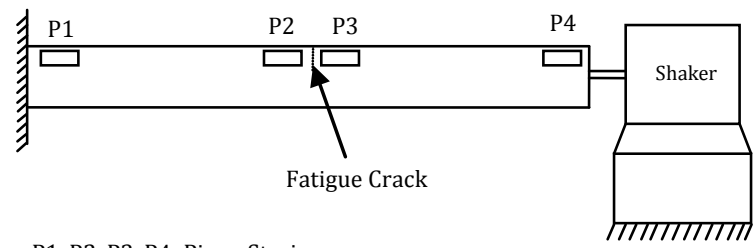

P1, P2, P3, P4: Piezo-Strain

Fig.8.Schematic of experimental setup

dynamic responses. Two of them were glued near the crack and out of other two, one is glued near the free end of the bar and other is glued near the fixed end of the bar. The frequency sweep was performed to obtain the resonant frequency of the bar. The cracked bar was excited then at this resonant frequency and the responses were obtained at the four piezo-strain gauges.

The magnitude of force vibration amplitude was $16 \mathrm{~N}$ for the given resonant frequency of excitation. These responses obtained were processed using picoscope taking into account Nyquist criteria to avoid aliasing error and Hanning window to minimize leakage error in the signal acquired.

From the frequency sweep it was found that the resonance occurs at the frequency of $2821 \mathrm{~Hz}$. At this 
resonant frequency, the cracked bar was excited. It was found that the responses obtained near the crack (Fig. 9(b) and Fig.9(c)) showed the presence of low frequency component, hamonics of resonant frequency of excitaion and nonlinear resonant frequency. The interaction of two

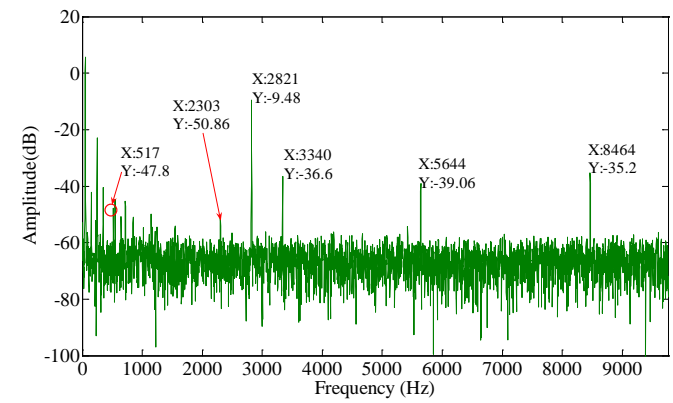

(a) Frequency response from sensor P1

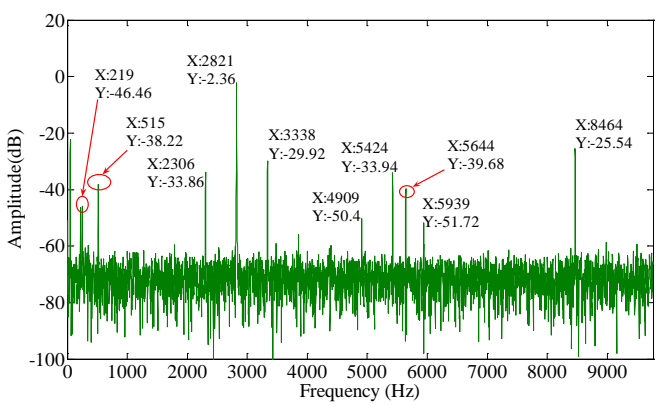

(b) Frequency response from sensor P2

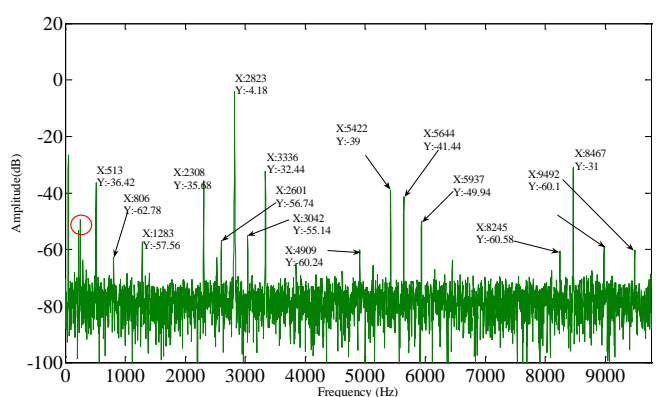

(c) Frequency response from sensor P3

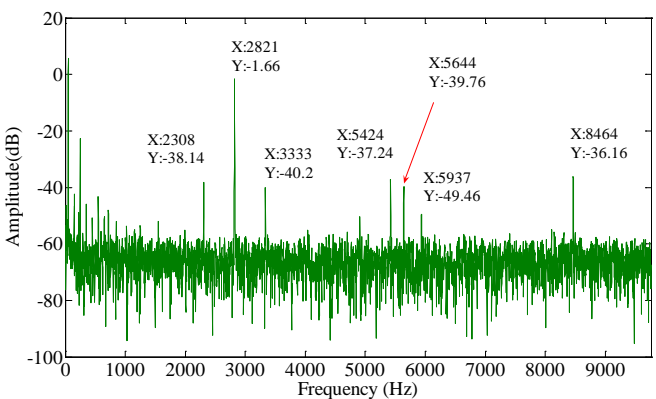

(d) Frequency response from sensor P4

Fig. 9. Response at resonant frequency for high excitation amplitude harmonic components leads to the modulation indicated by arrival of side band harmonic components. A nonlinear transformation of modulated signal by crack produce detection of low frequency component indicatedin numerical analysis. From the responses (Fig. 9(a) and Fig.9(d)) obtained far away from the crack it was found that responses were similar to those obtained near the crack but there was reduction in the amplitude of the component. This gives an indication that crack behave like an amplifier as the magnitude of the low frequency component is proportional to the magnitude of the bar response at frequency of excitation. From this it can be inferred that the crack behaves as a modulator, detector of low frequency component and an amplifier.

\section{Conclusion}

From the analytical model developed it can be found that the crack induced nonlinearity has a strong localised effect on the dynamics of the cracked bar. Simultaneously it transmits a low frequency component along the structure which is a result of complex nonlinear response including splitting of resonant frequencies, arrival of side bands and detection. This unique signature of a crack behaviour as modulator, detector and amplifier can be used for the structural health monitoring.

\section{References}

1. A. Dimarogonas, Vibration of cracked structures: A state of the art review, Engineering Fracture Mechanics,55,5 (1996)

2. V. Babitsky, Theory of vibro-impact systems and applications, 1998, Springer, Berlin (Revised translation from Russian, Nauka, Moscow, 1978).

3. I. Babakov, Theory of vibrations, Nauka, Moscow, 1968 (In Russian). 